\title{
Concomitant iGlarLixi and Sodium-Glucose Co- transporter-2 Inhibitor Therapy in Adults with Type 2 Diabetes: LixiLan-G Trial and Real-World Evidence Results
}

\author{
Cristian Guja (D) - Francesco Giorgino • Lawrence Blonde • \\ Amar Ali · Martin Prázný · Juris J. Meier · Elisabeth Souhami · \\ Robert Lubwama · Chen Ji · Julio Rosenstock
}

Received: September 3, 2021 / Accepted: November 1, 2021 / Published online: December 11, 2021

(C) The Author(s) 2021

\section{ABSTRACT}

Introduction: iGlarLixi, the once-daily fixedratio combination of insulin glargine $100 \mathrm{U} / \mathrm{ml}$ and lixisenatide, robustly improves glycaemic control in adults with type 2 diabetes irrespective of previous treatment [oral antihyperglycaemic drugs (OADs), basal insulin or glucagonlike peptide-1 receptor agonists (GLP-1 RAs)]. Sodium-glucose co-transporter-2 inhibitors (SGLT2is) are a recommended treatment option

Supplementary Information The online version contains supplementary material available at https:// doi.org/10.1007/s13300-021-01180-1.

\section{Guja $(\bowtie)$}

Department of Diabetes, Nutrition and Metabolic Diseases, Carol Davila University of Medicine and Pharmacy, Bucharest, Romania

e-mail: cristian.guja@b.astral.ro

\section{F. Giorgino}

Department of Emergency and Organ

Transplantation, Section of Internal Medicine, Endocrinology, Andrology and Metabolic Diseases, University of Bari Aldo Moro, Bari, Italy

\section{Blonde}

Department of Endocrinology, Ochsner Medical Center, Frank Riddick Diabetes Institute, New Orleans, LA, USA

\section{A. Ali}

Oakenhurst Medical Practice, Blackburn, UK for people with type 2 diabetes with cardiovascular disease, kidney disease and/or heart failure because of their cardio- and renoprotective benefits. Herein, we assessed the effects of concomitant iGlarLixi and SGLT2i therapy.

Methods: We conducted subgroup analyses according to SGLT2i use in: (1) adults with suboptimally controlled type 2 diabetes on GLP-1 RAs and OADs switching to iGlarLixi in the 26-week LixiLan-G randomised controlled trial (RCT; NCT02787551) and (2) adults switching to or adding iGlarLixi in a 6-month, retrospective real-world evidence (RWE) observational study using data from the US Optum-Humedica electronic medical records database. Changes in

M. Prázný

3rd Department of Internal Medicine, 1st Faculty of Medicine, Charles University in Prague, Prague,

Czech Republic

J. J. Meier

Diabetes Division, St Josef Hospital, Ruhr-University Bochum, Bochum, Germany

E. Souhami

Sanofi, Paris, France

R. Lubwama

Sanofi, Bridgewater, NJ, USA

C. Ji

Sanofi, Beijing, China

J. Rosenstock

Dallas Diabetes Research Center at Medical City, Dallas, TX, USA 
$\mathrm{HbA}_{1 \mathrm{c}}$ and hypoglycaemia prevalence and event rates were assessed.

Results: There were no major differences in baseline characteristics for those who initiated iGlarLixi while already using SGLT2i $(n=346)$ and those initiating iGlarLixi without concomitant SGLT2i therapy $(n=1285)$. $\mathrm{HbA}_{1 \mathrm{c}}$ reductions from baseline to time of assessment and hypoglycaemia prevalence and event rates were similar for iGlarLixi users regardless of SGLT2i therapy.

Conclusion: Evidence from an RCT and an RWE analysis supports the efficacy/effectiveness and safety of iGlarLixi when used concomitantly with SGLT2i.

Trial Registration: NCT02787551.

\section{PLAIN LANGUAGE SUMMARY}

People with type 2 diabetes require glucoselowering drugs to attain and maintain blood glucose control, with many eventually requiring injectable therapies. iGlarLixi combines two injectable therapies (basal insulin glargine and a glucagon-like peptide-1 receptor agonist, lixisenatide) into a single fixed-ratio daily injection and has previously been shown to provide robust improvements in blood glucose control. However, previous studies have not assessed the potential effect that simultaneous use of sodium-glucose co-transporter-2 inhibitor (SGLT2i) therapy may have on iGlarLixi therapy. This is of interest because SGLT2is are a widely used oral blood-glucose-lowering therapy that also have a beneficial effect in people with type 2 diabetes who have cardiovascular or kidney disease or have risk factors for the development or progression of these conditions. Therefore, this study assessed whether there were relevant differences in terms of blood glucose control and safety when initiating iGlarLixi in people treated with SGLT2i versus initiating iGlarLixi in people not receiving SGLT2i. Results showed that iGlarLixi provided similarly good glucose control and safety profiles, regardless of whether SGLT2is were used or not, thereby supporting the simultaneous use of these two therapies.
Keywords: Basal insulin; GLP-1 analogue; Glycaemic control; Hypoglycaemia; iGlarLixi; SGLT2 inhibitor; Type 2 diabetes

\section{Key Summary Points}

\section{Why carry out this study?}

iGlarLixi is a fixed-ratio combination of insulin glargine $100 \mathrm{U} / \mathrm{ml}$ and the glucagon-like peptide- 1 receptor agonist (GLP-1 RA), lixisenatide, which robustly improves glycaemic control in adults with type 2 diabetes irrespective of previous treatment (oral antihyperglycaemic drugs [OADs], basal insulin, or GLP-1 RAs).

However, the effects of concomitant iGlarLixi and sodium-glucose cotransporter-2 inhibitor (SGLT2i) therapy, a widely used OAD with known cardioand renoprotective benefits, have not been previously assessed.

Here, we aimed to explore the effects of concomitant SGLT2i use on the efficacy and safety of iGlarLixi in adults with type 2 diabetes using data from two sources: a post hoc exploratory analysis of a randomised controlled trial (RCT) - the LixiLan-G study-and real-world evidence from electronic medical records (EMRs) of individuals from the US OptumHumedica database.

\section{What has been learned from the study?}

The present analysis showed similar robust glycaemic outcomes and comparably low hypoglycaemia rates in people with suboptimally controlled type 2 diabetes who initiated iGlarLixi, irrespective of concomitant SGLT2i therapy.

In conclusion, evidence from an RCT and a real-world clinical setting support the efficacy/effectiveness and safety of iGlarLixi when used in combination with SGLT2i. 


\section{DIGITAL FEATURES}

This article is published with digital features, including an infographic, to facilitate understanding of the article. To view digital features for this article go to https://doi.org/10.6084/ m9.figshare.16913317.

\section{INTRODUCTION}

American Diabetes Association and European Association for the Study of Diabetes guidelines recommend that people with type 2 diabetes are regularly assessed and their therapy intensified if their $\mathrm{HbA}_{1 \mathrm{c}}$ targets are not reached $[1,2]$. First-line pharmacotherapy with metformin is recommended, followed by intensification with other antihyperglycaemic drugs, including sodium-glucose co-transporter-2 inhibitors (SGLT2is) or glucagon-like peptide-1 receptor agonists (GLP-1 RAs), if needed [1, 2]. Moreover, SGLT2is and/or GLP-1 RAs with proven cardioand renoprotective benefits are now recommended over other drug classes in people with type 2 diabetes who have established cardiovascular disease, kidney disease and/or heart failure (or risk indicators for these conditions), independent of current/target $\mathrm{HbA}_{1 \mathrm{c}}$ [3]. For those receiving SGLT2is and GLP-1 RAs who require additional glycaemic control, intensification with basal insulin (BI) is a recommended option, by either adding BI separately to the existing therapy or switching from their previous GLP-1 RA therapy to a fixed-ratio combination (FRC) of BI and GLP-1 RA [1, 2].

iGlarLixi, a once-daily injectable FRC of insulin glargine $100 \mathrm{U} / \mathrm{ml}$ (iGlar) and the GLP-1 RA lixisenatide (Lixi), is effective and well tolerated in people with type 2 diabetes suboptimally controlled by oral antihyperglycaemic drugs (OADs) alone or combined with $\mathrm{BI}$ or GLP-1 RA, as shown by the LixiLan-O, LixiLan-L and LixiLan-G randomised controlled trials (RCTs), respectively [4-6]. However, concomitant use of iGlarLixi and SGLT2is has not been specifically investigated.

Here, we present data on the efficacy/effectiveness and safety of concomitant iGlarLixi and SGLT2i use versus iGlarLixi use without
SGLT2i in adults with type 2 diabetes from two sources: a post hoc exploratory analysis of the LixiLan-G study and real-world evidence (RWE) from electronic medical records (EMRs) of individuals from the US Optum-Humedica database. The Optum-Humedica database includes EMR data for $>30$ million individuals [7].

\section{METHODS}

\section{Study Designs and Participants}

The design and methodology of the LixiLan-G study (NCT02787551) have been previously described [6]. In brief, LixiLan-G was a 26-week, randomised, open-label, active-controlled, parallel-group, Phase 3 study that aimed to evaluate the efficacy and safety of switching to iGlarLixi versus continuing treatment with prior GLP-1 RA therapy. Participants were adults with type 2 diabetes and suboptimal glycaemic control $\quad\left[\mathrm{HbA}_{1 \mathrm{c}}: \geq 7 \quad\right.$ to $\leq 9 \% \quad(\geq 53$ to $\leq 75 \mathrm{mmol} / \mathrm{mol}$ )] on OADs (metformin \pm pioglitazone \pm SGLT2i) and the maximum tolerated dose of a GLP-1 RA who were randomised (1:1) to switch to iGlarLixi or remain on their GLP-1 RA regimen with enforced adherence to therapy actively monitored throughout the study. iGlarLixi was injected subcutaneously once daily using one of two SoloSTAR (Sanofi, Paris, France) pen injectors according to the insulin dose required: for insulin doses of 10-40 U, a $2 \mathrm{U}$ iGlar: $1 \mu \mathrm{g}$ Lixi ratio pen (iGlarLixi doses of $10 \mathrm{U} / 5 \mu \mathrm{g}$ up to $40 \mathrm{U} / 20 \mu \mathrm{g}$ ) was used, and for insulin doses of 30-60 U, a $3 \mathrm{U}$ iGlar: $1 \mu \mathrm{g}$ Lixi ratio pen (iGlarLixi doses of $30 \mathrm{U} / 10 \mu \mathrm{g}$ up to $60 \mathrm{U} / 20 \mu \mathrm{g}$ ) was used. For people already using SGLT2is prior to randomisation, SGLT2i use was continued at the same dose regimen as prior to randomisation, with doses administered according to local product labelling. The LixiLan-G trial was designed and monitored in accordance with Good Clinical Practice guidelines, the International Conference on Harmonisation and the Declaration of Helsinki. Institutional review boards or ethics committees at each study site approved the protocol 
and all participants provided informed consent. Electronic healthcare records data are anonymised.

Additionally, a retrospective, observational cohort study (subsequently referred to as the RWE study) captured EMR data from the Optum-Humedica database for people who initiated iGlarLixi in a clinical practice setting between 1 January 2017 and 30 September 2018 in the US. Data on the type of pen used were not captured, but only the 3:1 ratio pen is licenced for use in the US [8]. Apart from iGlarLixi initiation, there were no prespecified inclusion or exclusion criteria related to diabetes medication. The index date was defined as the date of the first iGlarLixi prescription; the baseline period was defined as 180 days prior to and including the index date, and the follow-up period was defined as 180 days after the index date. Eligible participants were $\geq 18$ years of age with a diagnosis of type 2 diabetes, $\mathrm{HbA}_{1 \mathrm{c}} \geq 7 \%$ within 90 days prior to and 14 days after the index date, and $\geq 1 \mathrm{HbA}_{1 \mathrm{c}}$ result 75 to 180 days after iGlarLixi initiation. Permission to access and use data from the Optum-Humedica electronic medical records database was obtained.

\section{Outcomes}

The main efficacy endpoint in the post hoc exploratory analysis of LixiLan-G was change in $\mathrm{HbA}_{1 \mathrm{c}}$ from baseline to Week 26. Other endpoints included changes in fasting plasma glucose (FPG), 2-h post-prandial plasma glucose (2-h PPG), body weight and iGlarLixi dose, from baseline to Week 26. Safety endpoints included the prevalence and event rates of documented symptomatic hypoglycaemia $[\leq 3.9 \mathrm{mmol} / \mathrm{l}$ $(\leq 70 \mathrm{mg} / \mathrm{dl})]$ or severe symptomatic hypoglycaemia (event requiring assistance of another person to actively administer carbohydrate, glucagon or other resuscitative actions) and treatment-emergent adverse events (TEAEs).

In the RWE study, the effectiveness of concomitant iGlarLixi and SGLT2i therapy was assessed based on $\mathrm{HbA}_{1 \mathrm{c}}$ change from baseline to Month 6 . The main safety parameters assessed were the prevalence and event rates of any hypoglycaemia or hypoglycaemia requiring inpatient admission or an emergency department (ED) visit. Other safety parameters were diabetic ketoacidosis (DKA), acute kidney injury (AKI) requiring hospitalisation and urinary tract infections (UTI), including severe complications requiring hospitalisation or an ED visit for pyelonephritis or urosepsis. Data extraction was performed by a trained analyst and reviewed by an independent analyst to validate coding rules, order of operations and statistical methodology at each step. Data were stored in a Medidata Rave database and any modification in the database was traced using an audit trail.

\section{Data analysis and Statistics}

For the LixiLan-G study, the sample size calculations were based on the primary efficacy endpoint (change in $\mathrm{HbA}_{1 \mathrm{c}}$ from baseline to week 26), a common standard deviation [SD] of $1.1 \%$, a $0.4 \%$ mean difference between iGlarLixi and GLP-1 RA in change in $\mathrm{HbA}_{1 \mathrm{c}}$, an estimated dropout rate of $20 \%$ and a $t$-test at a two-sided $5 \%$ significance level with at least $90 \%$ power [6]. On that basis, 500 participants were required to be included for the study to be appropriately powered and LixiLan-G randomised 514 participants [6]. The RWE study was observational and descriptive. All those who met the eligibility criteria in the database were included in the analysis.

In the LixiLan-G subgroup analysis, a mixedeffect model with repeated measures (MMRM) approach was used to assess changes from baseline to Week 26 in $\mathrm{HbA}_{1 \mathrm{c}}$, FPG and body weight across SGLT2i user and non-user subgroups, while change in 2-h PPG was analysed using an analysis of covariance (ANCOVA) model. The MMRM model included treatment groups, randomisation strata of $\mathrm{HbA}_{1 \mathrm{c}}$ $(<8.0 \%, \geq 8.0 \%)$ at Visit 1, GLP-1 RA subtype at screening, scheduled visit, SGLT2i use, world region, treatment-by-visit, treatment-by-SGLT2i use, visit-by-SGLT2i use, treatment-by-visit-bySGLT2i use and baseline value-by-visit interaction. The ANCOVA model included treatment groups, randomisation strata of $\mathrm{HbA}_{1 \mathrm{c}}$ $(<8.0 \%, \geq 8.0 \%)$ at Visit 1, GLP-1 RA subtype at screening, SGLT2i use, treatment-by-SGLT2i 
use, world region and baseline value of the covariate being tested. Safety analyses were performed using descriptive statistics.

In the RWE study, descriptive statistics were used to describe baseline characteristics, effectiveness and safety endpoints in participants who initiated iGlarLixi with concomitant SGLT2i therapy compared with those who initiated iGlarLixi without SGLT2i use.

\section{RESULTS}

\section{Participant Disposition and Baseline Characteristics}

Among those randomised to iGlarLixi in the LixiLan-G subgroup analysis, there were 26 SGLT2i users and 231 SGLT2i non-users. At baseline, SGLT2i users tended to have a lower body mass index and a longer duration of diabetes than SGLT2i non-users. Other demographic and disease characteristics were generally similar between the two SGLT2i subgroups (Supplementary Table 1).

In the RWE study, 320 participants who received iGlarLixi were SGLT2i users and 1054 participants were SGLT2i non-users. At baseline, SGLT2i users tended to be younger and have a lower $\mathrm{HbA}_{1 \mathrm{c}}$ than SGLT2i non-users. Other demographic and disease characteristics were generally similar between the two SGLT2i subgroups (Supplementary Table 1).

$\mathrm{HbA}_{1 \mathrm{c}}$

In both the LixiLan-G and RWE analyses, $\mathrm{HbA}_{1 \mathrm{c}}$ changes were similar overall between SGLT2i users and non-users (Fig. 1). In LixiLan-G, mean (SD) baseline $\mathrm{HbA}_{1 \mathrm{c}}$ was $7.8 \quad(0.7) \%$ (61.2 [7.2] $\mathrm{mmol} / \mathrm{mol})$ and $7.8(0.6) \%$ (61.5 [6.9] $\mathrm{mmol} / \mathrm{mol}$ ) for SGLT2i users and non-users, respectively. The least-squares (LS) mean (standard error $[\mathrm{SE}]$ ) change in $\mathrm{HbA}_{1 \mathrm{c}}$ from baseline to Week 26 was - $1.15 \quad(0.16) \%$ $(-12.6[1.7] \mathrm{mmol} / \mathrm{mol})$ in the SGLT2i users and $-1.01(0.05) \%(-11.0[0.5] \mathrm{mmol} / \mathrm{mol})$ in the non-users.
In the RWE study, mean (SD) baseline $\mathrm{HbA}_{1 \mathrm{c}}$ was $9.2(1.6) \%(76.8[17.2] \mathrm{mmol} / \mathrm{mol})$ and $9.5(1.7) \%(80.4$ [18.5] $\mathrm{mmol} / \mathrm{mol})$ for SGLT2i users and non-users, respectively. Mean (SD) change in $\mathrm{HbA}_{1 \mathrm{c}}$ from baseline to Month 6 was $-0.89(1.57) \%(-9.7[17.2] \mathrm{mmol} / \mathrm{mol})$ in SGLT2i users and $-1.05 \quad(1.85) \% \quad(-11.5$ [20.2] $\mathrm{mmol} / \mathrm{mol}$ ) in non-users.

\section{Other Efficacy Endpoints-LixiLan-G Data}

In the LixiLan-G subgroup analysis, the changes in FPG and 2-h PPG from baseline to Week 26 were also similar between the SGLT2i subgroups (Fig. 1a). A small numerical increase in mean body weight was observed from baseline to Week 26 in those initiating iGlarLixi, irrespective of SGLT2i use (SGLT2i users: LS mean change [SE] 2.4 [0.6] kg; SGLT2i non-users: LS mean change [SE] 1.9 [0.2] kg).

At Week 26, there was no difference in mean \pm SD daily iGlarLixi dose between SGLT2i users (iGlar $42.0 \pm 14.9 \mathrm{U}$; Lixi $16.1 \pm 3.9 \mu \mathrm{g}$ ) and SGLT2i non-users (iGlar $43.7 \pm 15.1 \mathrm{U}$; Lixi $16.6 \pm 3.8 \mu \mathrm{g})$.

\section{Safety}

\section{Hypoglycaemia}

In the LixiLan-G subgroup analysis, the percentage of participants with documented symptomatic hypoglycaemia [plasma glucose $\leq 3.9 \mathrm{mmol} / \mathrm{l} \quad(\leq 70 \mathrm{mg} / \mathrm{dl})]$ was similar between the SGLT2i subgroups $(24.0 \%$ and $28.3 \%$ in SGLT2i users and non-users, respectively, Table 1, part a). The corresponding number of events per participant-year (PPY) tended to be lower in the SGLT2i users (0.72) than in the SGLT2i non-users (1.62). Only one severe hypoglycaemia event was recorded, affecting a participant in the SGLT2i non-user subgroup.

In the RWE study, the percentage of participants with any hypoglycaemia at Month 6 was similar in SGLT2i users (7.5\%) and SGLT2i nonusers $(6.7 \%)$ (Table 1 , part b). The corresponding event rate PPY was also similar between SGLT2i users (0.26) and SGLT2i non-users (0.24). The proportion of participants with 


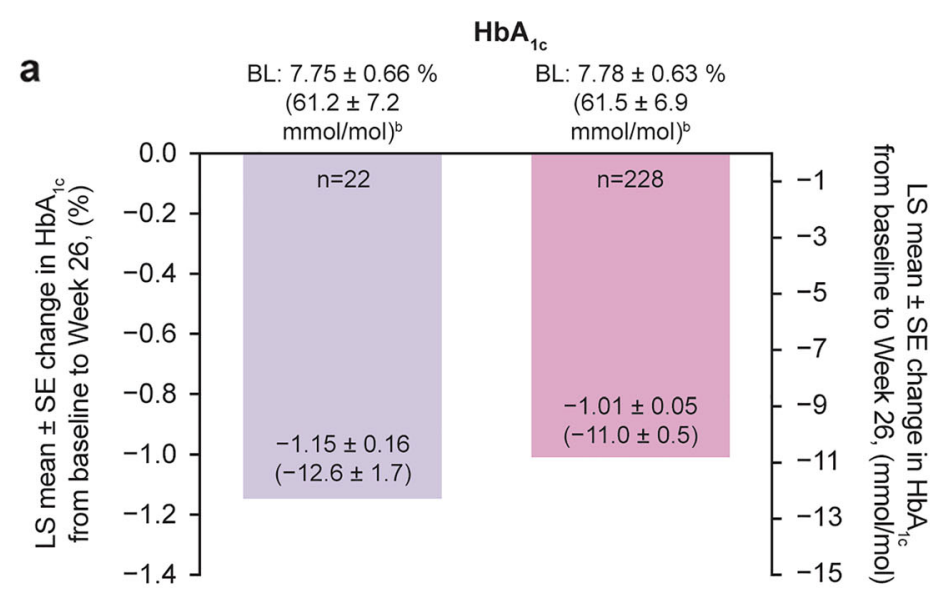

FPG

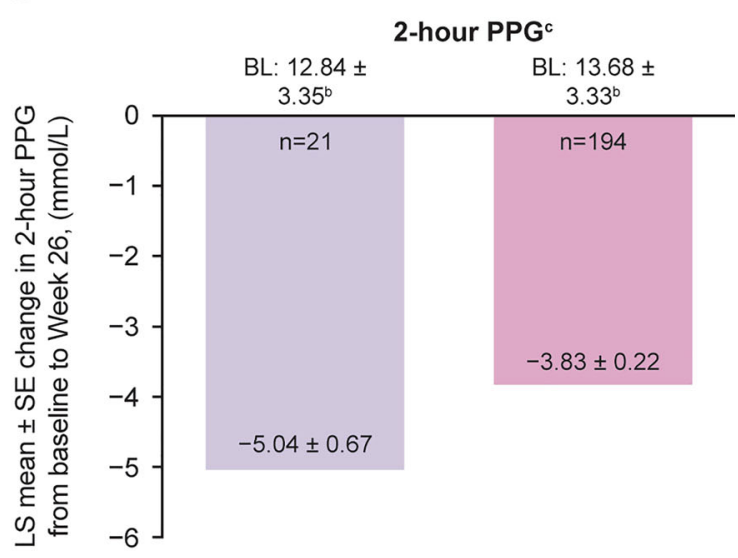

b

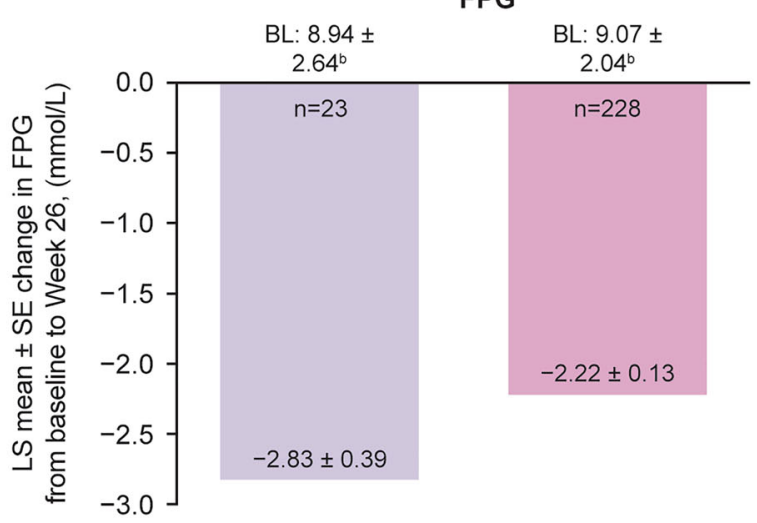

$\mathrm{HbA}_{1 \mathrm{c}}$

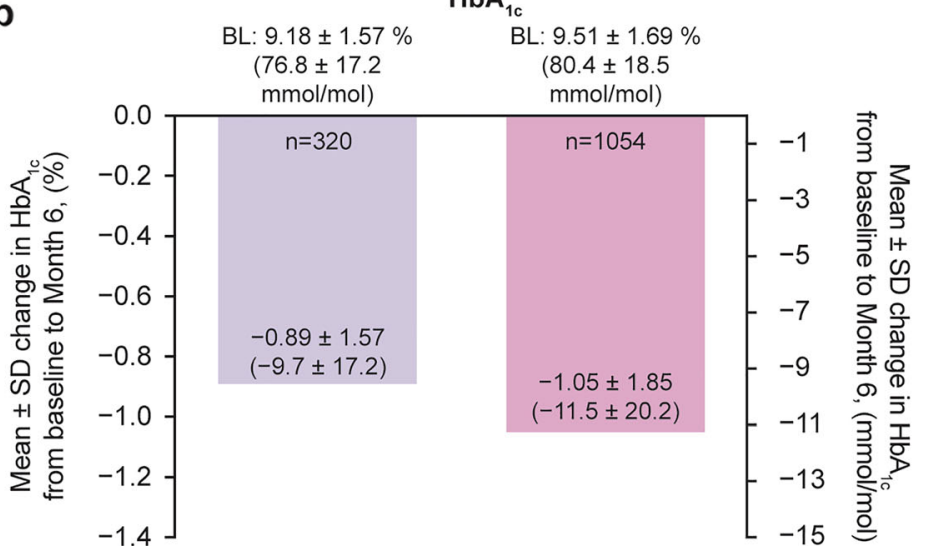

iGlarLixi; SGLT2i users

iGlarLixi; SGLT2i non-users

Fig. 1 Glycaemic control results by study: a LixiLan-G subgroup analysis ${ }^{a}$, b RWE study. ${ }^{a}$ All participants in LixiLan-G were also receiving metformin; all data are presented for the mITT population, defined as all randomised participants with a baseline assessment and at least one post-baseline assessment for any primary or secondary efficacy variables. ${ }^{b}$ Baseline data are presented as glucose; iGlarLixi a fixed-ratio combination of insulin glargine $100 \mathrm{U} / \mathrm{ml}$ and the glucagon-like peptide-1 receptor agonist, lixisenatide; $L S$ least squares; $S D$ standard deviation; $S E$ standard error; $m I T T$ modified intent to treat; $P P G$ post-prandial plasma glucose; $R W E$ real-world evidence; $\quad S G L T 2 i$ sodium-glucose co-transporter-2 inhibitor standardised meal test. BL baseline, FPG fasting plasma 


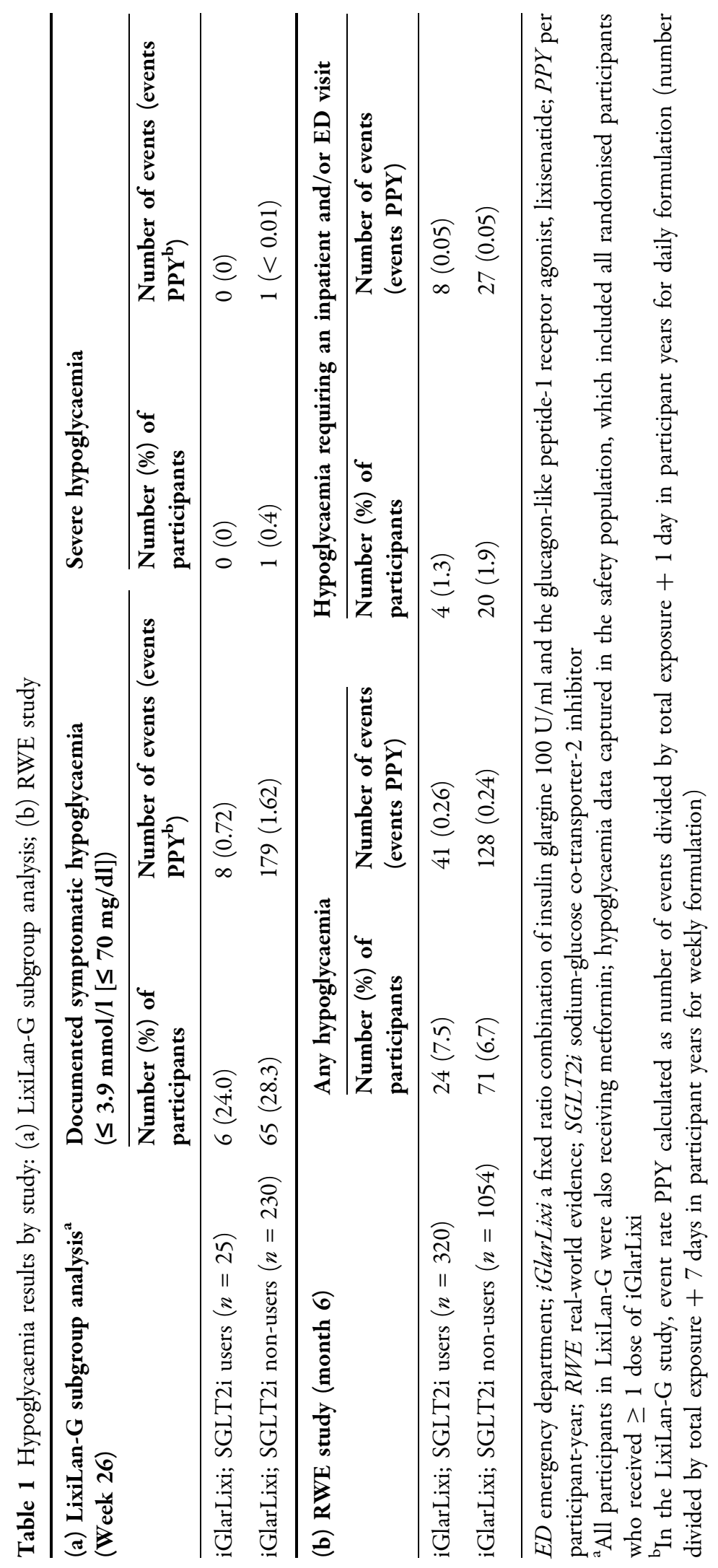


hypoglycaemia events associated with an inpatient or ED visit were also similar between the SGLT2i subgroups (SGLT2i users: 1.3\%; SGLT2i non-users: $1.9 \%$ ), as were the corresponding event rates PPY (SGLT2i users: 0.05; SGLT2i non-users: 0.05 ).

\section{Other Safety Endpoints}

In the LixiLan-G subgroup analysis, the results for the safety endpoints were generally similar between the SGLT2i subgroups at Week 26. In the SGLT2i user group, there were no TEAEs leading to permanent treatment discontinuation, while nine $(3.9 \%)$ participants in the SGLT2i non-user subgroup had such an event (Supplementary Table 2).

In the RWE study, the proportions of participants with DKA, AKI and UTIs at 6-month follow-up were very low and similar between SGLT2i users and SGLT2i non-users (Supplementary Table 3 ).

Descriptive analyses of the RWE study showed that the proportion of participants with any-cause hospitalisation or ED visit appeared to be slightly lower for the SGLT2i users than the non-users at both baseline and 6-month follow-up (Supplementary Table 4).

\section{DISCUSSION}

The present post hoc analysis of the LixiLan-G RCT and the RWE assessment of US EMRs from the Optum-Humedica database both showed similar robust glycaemic outcomes and comparably low hypoglycaemia rates in people with suboptimally controlled type 2 diabetes who initiated iGlarLixi, irrespective of concomitant SGLT2i therapy. In the LixiLan-G RCT, participants in the SGLT2i users subgroup were already on stable treatment with SGLT2i, metformin and GLP-1 RAs and were suboptimally controlled on this treatment. Switching to iGlarLixi provided overall similar results in these participants compared with those who were suboptimally controlled on GLP-1 RAs and metformin only (SGLT2i non-users). In the RWE study, there were no eligibility criteria relating to medication beyond initiating iGlarLixi; however, baseline demographics show that other medication use was similar between SGLT2i users and non-users. As such, the impact of SGLT2i treatment in the users' group was already accounted for in their baseline glucose values, providing that they had not recently initiated SGLT2i therapy. This may explain why the addition of iGlarLixi was not associated with an additional effect on glycaemic control in the SGLT2i users group versus the non-user group. These findings are particularly relevant given that use of SGLT2i therapy has increased in recent years and a significant future increase is anticipated $[9,10]$. While there was a difference in mean baseline $\mathrm{HbA}_{1 \mathrm{c}}$ between the RCT and RWE studies, this was not surprising as $\mathrm{HbA}_{1 \mathrm{c}}$ values are usually higher in RWE studies compared with RCTs.

The efficacy and safety of iGlarLixi have also been demonstrated in Japanese populations permitted to include SGLT2 $\mathrm{i}$ as a concomitant medication [11, 12]. Furthermore, the finding that iGlarLixi is as effective and safe when used concomitantly with SGLT2i versus without SGLT2i is consistent with data for the other available FRC, insulin degludec and liraglutide (IDegLira). The efficacy and safety profile of IDegLira shown in the DUAL IX study [13], in which all participants used SGLT2i, was comparable to that observed in other DUAL studies in participants who were not receiving SGLT2i $[14,15]$.

Study limitations included the post hoc nature of the LixiLan-G analysis and the inherent issues associated with RWE data, such as missing information (including on use of background or concomitant medications) and potential unknown confounders and bias. An additional possible limitation of the study is the small number of LixiLan-G participants taking an SGLT2i, along with the smaller population of SGLT2i-users than non-users in the Optum-Humedica database; this is likely due to the low overall use of SGLT2i in clinical practice during the period when the studies were conducted. There was also potential under-recognition of hypoglycaemia in the RWE study. The strength of this analysis is the complementary nature of the data provided by an RCT and RWE study supporting both efficacy and effectiveness. However, comparisons between these two 
studies should be cautious and considered in the context of the different population characteristics, including different degrees of metabolic control at baseline (due to more restrictive eligibility criteria in the LixiLan-G RCT) and the higher degree of participant follow-up in the LixiLan-G trial compared with the real-world data as well as overall differences in the management of these populations.

\section{CONCLUSIONS}

In conclusion, results of the present analysis, which combine evidence from an RCT and a real-world clinical setting, showed that iGlarLixi provided similar robust glycaemic control and comparably low risk of hypoglycaemia in people with type 2 diabetes regardless of concomitant use of SGLT2i, thereby supporting the combined use of iGlarLixi and SGLT2i.

\section{ACKNOWLEDGEMENTS}

We thank the study participants, trial staff and investigators for their participation. We also thank Ana Merino-Trigo, PhD (Sanofi), for coordinating the development, facilitating author discussions and review of this manuscript.

Funding. The LixiLan-G RCT and RWE studies were sponsored by Sanofi. The Rapid Service publication fee was funded by Sanofi.

Medical writing and Editorial Assistance. Editorial and writing assistance was provided by Cameron Hubert, $\mathrm{PhD}$, and Tamsin Brown, MSc, of Fishawack Communications, Ltd., part of Fishawack Health, UK, and was funded by Sanofi.

Authorship. All named authors meet the International Committee of Medical Journal Editors (ICMJE) criteria for authorship of this article, take responsibility for the integrity of the work as a whole, and have given their approval for this version to be published.
Authors' Contributions. Robert Lubwama and Chen Ji contributed to the study design and conduct/data collection and analysis. All authors contributed to the writing and interpretation of results and gave final approval of the version to be published.

Prior Presentation. Selected data from this manuscript were presented as a poster at the 80th Virtual Scientific Sessions of the American Diabetes Association, June 12-16 2020.

Disclosures. Cristian Guja has participated in scientific advisory boards for and received consulting fees from AstraZeneca, Boehringer Ingelheim, Eli Lilly, MSD, Novo Nordisk, Sanofi and Servier. Francesco Giorgino has participated in scientific advisory boards and received consulting fees from AstraZeneca, Boehringer Ingelheim, Eli Lilly, MSD, Novo Nordisk, Roche Diabetes Care and Sanofi. Lawrence Blonde declares grant/research support to himself and/ or his institution from: Janssen Pharmaceuticals, Inc., Lexicon Pharmaceuticals, Inc., Merck \& Co., Novo Nordisk and Sanofi; speaker for: Janssen Pharmaceuticals, Inc., Novo Nordisk and Sanofi; and consultant for: AstraZeneca, Corcept Therapeutics, Gilead Sciences, Inc., Janssen Pharmaceuticals, Inc., Lyndra Therapeutics, Merck \& Co., Novo Nordisk, Salix Pharmaceuticals and Sanofi. Amar Ali has received research support and honoraria from Amgen, Eli Lilly, Gelesis, NAPP, Novartis, Novo Nordisk and Sanofi. Martin Prázný has received consulting and speaker's fees from Abbott, AstraZeneca, Boehringer Ingelheim, Eli Lilly, Medtronic, Novartis, Novo Nordisk, Sanofi, Takeda and Teva. Juris J. Meier has participated in scientific advisory boards for and received speaker's fees from AstraZeneca, Boehringer Ingelheim, Eli Lilly, MSD, Novo Nordisk, Sanofi and Servier; and has received research support from Boehringer Ingelheim, MSD, Novo Nordisk and Sanofi. Elisabeth Souhami, Robert Lubwama, and Chen Ji are employees of Sanofi. Julio Rosenstock - has served on advisory panels for Applied Therapeutics, Boehringer Ingelheim, Eli Lilly, Intarcia, Janssen, Lexicon, Novo Nordisk, Oramed and Sanofi; and has received research support from AstraZeneca, 
Boehringer Ingelheim, Eli Lilly, Genentech, GlaxoSmithKline, Intarcia, Janssen, Lexicon, Merck, Novo Nordisk, Oramed, Pfizer and Sanofi.

Compliance with Ethics Guidelines. The current analysis uses data from a previously published clinical trial and from electronic healthcare records from the Optum-Humedica electronic medical records database. Permission to access and use data from the Optum-Humedica electronic medical records database was obtained. The LixiLan-G trial was designed and monitored in accordance with Good Clinical Practice guidelines, the International Conference on Harmonisation and the Declaration of Helsinki. Institutional review boards or ethics committees at each study site approved the protocol and all participants provided informed consent. Electronic healthcare records data are anonymised.

Data Availability. The datasets generated during and/or analysed during the current study are available from the corresponding author on reasonable request.

Open Access. This article is licensed under a Creative Commons Attribution-NonCommercial 4.0 International License, which permits any non-commercial use, sharing, adaptation, distribution and reproduction in any medium or format, as long as you give appropriate credit to the original author(s) and the source, provide a link to the Creative Commons licence, and indicate if changes were made. The images or other third party material in this article are included in the article's Creative Commons licence, unless indicated otherwise in a credit line to the material. If material is not included in the article's Creative Commons licence and your intended use is not permitted by statutory regulation or exceeds the permitted use, you will need to obtain permission directly from the copyright holder. To view a copy of this licence, visit http://creativecommons.org/licenses/by$\mathrm{nc} / 4.0 /$.

\section{REFERENCES}

1. Davies MJ, D'Alessio DA, Fradkin J, et al. Management of hyperglycemia in type 2 diabetes, 2018. A consensus report by the American Diabetes Association (ADA) and the European Association for the Study of Diabetes (EASD). Diabetes Care. 2018. https://doi.org/10.2337/dci18-0033.

2. American Diabetes Association. 9. Pharmacologic approaches to glycemic treatment: standards of medical care in diabetes-2021. Diabetes Care. 2021;44:S111-24.

3. American Diabetes Association. 10. Cardiovascular disease and risk management: standards of medical care in diabetes-2021. Diabetes Care. 2021;44: S125-50.

4. Rosenstock J, Aronson R, Grunberger G, et al. Benefits of LixiLan, a titratable fixed-ratio combination of insulin glargine plus lixisenatide, versus insulin glargine and lixisenatide monocomponents in type 2 diabetes inadequately controlled on oral agents: the LixiLan-O randomized trial. Diabetes Care. 2016;39:2026-35.

5. Aroda VR, Rosenstock J, Wysham C, et al. Efficacy and safety of LixiLan, a titratable fixed-ratio combination of insulin glargine plus lixisenatide in type 2 LixiLan-L diabetes inadequately controlled on basal insulin and metformin: the randomized trial. Diabetes Care. 2016;39:1972-80.

6. Blonde L, Rosenstock J, Del Prato S, et al. Switching to iGlarLixi versus continuing daily or weekly GLP$1 \mathrm{RA}$ in type 2 diabetes inadequately controlled by GLP-1 RA and oral antihyperglycemic therapy: the LixiLan-G randomized clinical trial. Diabetes Care. 2019;42:2108-16.

7. Optum. Optum Integrated Data. Eden Prairie, MN. 2014. https://www.optum.com/content/dam/ optum3/optum/en/resources/fact-sheets/ Integrated-Data-product-sheet.pdf. Accessed 15 Oct 2021.

8. Sanofi. Soliqua ${ }^{\circledR}$ : US prescribing information. 2019. http://products.sanofi.us/soliqua100-33/ soliqua100-33.pdf. Accessed 30 June 2020.

9. Ni L, Yuan C, Chen G, Zhang C, Wu X. SGLT2i: beyond the glucose-lowering Effect. Cardiovasc Diabetol. 2020;19:98.

10. Scheen AJ. Sodium-glucose cotransporter type 2 inhibitors for the treatment of type 2 diabetes mellitus. Nat Rev Endocrinol. 2020;16:556-77.

11. Terauchi Y, Nakama T, Spranger R, Amano A, Inoue T, Niemoeller E. Efficacy and safety of insulin 
glargine/lixisenatide fixed-ratio combination (iGlarLixi 1:1) in Japanese patients with type 2 diabetes mellitus inadequately controlled on oral antidiabetic drugs: a randomized, 26-week, openlabel, multicenter study: the LixiLan JP-O2 Randomized Clinical Trial. Diabetes Obes Metab. 2020;22(Suppl 4):14-23.

12. Watada H, Takami A, Spranger R, Amano A, Hashimoto Y, Niemoeller E. Efficacy and safety of 1:1 fixed-ratio combination of insulin glargine and lixisenatide versus lixisenatide in japanese patients with type 2 diabetes inadequately controlled on oral antidiabetic drugs: the LixiLan JP-O1 randomized clinical trial. Diabetes Care. 2020;43:1249-57.

13. Philis-Tsimikas A, Billings L, Busch R, et al. Superior efficacy of insulin degludec/liraglutide versus insulin glargine U100 as add-on to sodium-glucose co- transporter-2 inhibitor therapy: a randomized clinical trial in people with uncontrolled type 2 diabetes. Diabetes Obes Metab. 2019;21:1399-408.

14. Gough SC, Bode B, Woo V, et al. Efficacy and safety of a fixed-ratio combination of insulin degludec and liraglutide (IDegLira) compared with its components given alone: results of a phase 3 , open-label, randomised, 26-week, treat-to-target trial in insulin-naive patients with type 2 diabetes. Lancet Diabetes Endocrinol. 2014;2:885-93.

15. Linjawi S, Bode BW, Chaykin LB, et al. The efficacy of IDegLira (insulin degludec/liraglutide combination) in adults with type 2 diabetes inadequately controlled with a GLP-1 receptor agonist and oral therapy: DUAL III randomized clinical trial. Diabetes Ther. 2017;8:101-14. 